\title{
Lentivirus-mediated knockdown of chondroitin polymerizing factor inhibits glioma cell growth in vitro
}

\author{
YANG-HUA FAN* ${ }^{*}$ BING XIAO* ${ }^{*}$ SHI-GANG LV, MIN-HUA YE, XIN-GEN ZHU and MIAO-JING WU \\ Department of Neurosurgery, The Second Affiliated Hospital of Nanchang University, Nanchang, Jiangxi 330006, P.R. China
}

Received November 15, 2016; Accepted May 26, 2017

DOI: $10.3892 / o r .2017 .5731$

\begin{abstract}
Glioma is the most common primary tumor in the central nervous system, characterized by rapid progression, aggressive behavior, frequent recurrence and poor prognosis. In the present study we demonstrated that chondroitin polymerizing factor (CHPF) is highly expressed in human glioma tissues and 4 glioma cell lines. To explore the role of $\mathrm{CHPF}$ in glioma, a lentiviral vector expressing CHPF shRNA was constructed and transfected into the glioma U251 cells, which stably downregulated the expression levels of the CHPF gene in U251 cells in vitro. U251 cell proliferation inhibition rates were determined by MTT assay. The effect of survivin shRNA on U251 cell cycle distribution and cell apoptosis was determined by flow cytometry. Compared to the shRNA-Ctrl group of cells, the shRNA-CHPF group of cells exhibited decreased proliferation and a significant increase in the proportion of cells in the G0/G1 phase. In addition, we found that knockdown of the expression of CHPF increased apoptosis in glioma U251 cells. Therefore, our results confirmed that CHPF promotes growth and inhibits apoptosis in glioma U251 cells. Thus, by in vivo and in vitro data, the present study suggests that CHPF could be a new potential therapeutic target for glioma.
\end{abstract}

\section{Introduction}

In adults, glioma is the most common type of malignant tumor of the brain and central nervous system, characterized by rapid progression, aggressive behavior, frequent recurrence and poor prognosis $(1,2)$. Despite advances in diagnosis and treatment, frequent recurrence and a low cure rate of glioma remain due to the rapid proliferation and high invasiveness of this disease, particularly for glioblastoma multiforme patients $(3,4)$. It is in essence a genetic disease with overexpression of oncogenes

Correspondence to: Dr Miao-Jing Wu or Dr Xin-Gen Zhu, Department of Neurosurgery, The Second Affiliated Hospital, Nanchang University, 1 Minde Road, Nanchang, Jiangxi 330006, P.R. China

E-mail: 905327378@qq.com

E-mail: xingenzhu_2015@sina.com

${ }^{*}$ Contributed equally

Key words: CHPF, glioma, proliferation, apoptosis and deletions or mutations of tumor-suppressor genes, which lead to the uncontrolled proliferation of cancer cells $(5,6)$. Therefore, it is essential to investigate the underlying mechanisms of this disease.

A human cDNA encoding the novel protein chondroitin polymerizing factor (CHPF) which was initially discovered by Kitagawa et al in 2003, is essential for chondroitin polymerizing activity (7). In addition, knockdown of the expression of CHPF leads to specific elimination of chondroitin sulfate (CS) and dermatan sulfate. In recent years, more and more evidence has revealed the potential roles of CS in tumor biology. Notably, numerous studies have shown that the CS expression has been observed significantly increased in many human tumors, such as colon (8) and ovarian epithelial cancer (9). The gene encoding CHPF has been identified in humans (10) and mice (11). Thus, CHPF plays an important role in tumor progression. However, no studies have been carried out investigating the functional role of CHPF in human types of cancer, and particularly in glioma.

In the present study, we demonstrated that CHPF was highly expressed in human glioma tissues and 4 glioma cell lines. Consequently, we constructed a lentivirus vector mediating RNAi targeting of CHPF (LV-shRNA-CHPF). We employed the LV-shRNA-CHPF to determine whether CHPF affects the progression of human glioma in vitro.

\section{Materials and methods}

Cancer cell lines. The human glioma cell lines A172, U373, U251, U87 and the human renal epithelial 293T cells were purchased from the Shanghai Cell Bank (Shanghai, China). The cells were cultured in Dulbecco's modified Eagle's medium (DMEM) (Gibco, Carlsbad, CA, USA) containing $10 \%$ fetal bovine serum (Invitrogen, Carlsbad, CA, USA) in an incubator at $37^{\circ} \mathrm{C}$ with $5 \% \mathrm{CO}_{2}$.

Patients and tissue samples. Patients with glioma $(\mathrm{n}=32)$ who underwent initial surgery at The Second Affiliated Hospital of Nanchang University between 2013 and 2015 were retrospectively selected for the present study. Normal brain tissues $(n=10)$ were obtained from epileptic resections. Each patient participated after providing informed consent, and the use of the samples for research was approved by the Medical Ethics Committee of The Second Affiliated Hospital of Nanchang University. All specimens were immediately frozen in liquid 
nitrogen after resection and stored at $-80^{\circ} \mathrm{C}$ until RNA extraction. No patients had received therapy before resection.

Quantitative real-time polymerase chain reaction ( $q R T-P C R$ ) assay. Total RNA was isolated from the 4 glioma cell lines (A172, U373, U251 and U87) using TRIzol Total RNA Reagent (Invitrogen, Shanghai, China). Complementary DNA (cDNA) synthesis was performed with $2 \mu \mathrm{g}$ of total RNA using the RevertAid ${ }^{\mathrm{TM}} \mathrm{H}$ Minus First Strand cDNA Synthesis kit (Takara, Otsu, Japan). The CHPF primers were obtained from GeneChem Co. Ltd. (Shanghai, China), and GAPDH was used as an internal control. The primers used were as follows: CHPF forward, 5'-GGA ACG CAC GTA CCA GGAG-3' and reverse, 5'-CAC CCT GTT GCT GTA GCC AAA-3'; and GAPDH forward, 5'-TGA CTT CAA CAG CGA CAC CCA-3' and reverse, 5'-CAC CCT GTT GCT GTA GCC AAA-3'. Quantitative PCR was performed using the SYBR PrimeScript RT-PCR kit (Takara) on an Applied Biosystems 7300 fluorescence quantitative PCR system (Applied Biosystems, Foster City, CA, USA). The reaction mixtures were incubated at $95^{\circ} \mathrm{C}$ for $30 \mathrm{sec}$, followed by 45 amplification cycles at $95^{\circ} \mathrm{C}$ for $5 \mathrm{sec}$ and $60^{\circ} \mathrm{C}$ for $30 \mathrm{sec}$. The PCR products of CHPF and GAPDH were 117 and $121 \mathrm{bp}$, respectively. The relative expression of the CHPF mRNA was calculated with the $2^{-\Delta \Delta \mathrm{Ct}}$ method, using the GAPDH mRNA expression level for normalization.

Construction of the shRNA lentiviral vector and cell transfection. The lentiviral expressing the short hairpin RNA (shRNA) targeting the sequence of the CHPF gene (CTGGCCATGCT ACTCTTTG) and negative control (NC) (TTCTCCGAACGT GTCACGT) were purchased from Shanghai GeneChem Co., Ltd. Then, they were cloned into the pGCSIL-GFP lentiviral vector with $A g e I / E c o R I$ sites to form the recombinant lentiviral shRNA expression vector. Lentiviral vectors and packaging vectors were transfected into $293 \mathrm{~T}$ cells using Lipofectamine 2000. Lentiviral particles were purified using ultracentrifugation and the titer of the lentiviruses was determined by endpoint dilution assay.

The U251 glioblastoma cells were infected with the shRNA-CHPF-lentivirus or the NC lentivirus. The U251 cells were seeded in a 6 -well plate at $40 \%$ confluence with $4.0 \times 10^{5}$ cells/well. After $72 \mathrm{~h}$ of infection, the cells were observed under a fluorescence microscope (MicroPublisher 3.3RTV; Olympus, Tokyo, Japan). After 5 days of infection, the ability of the shRNA-CHPF vectors to knock down CHPF was investigated using quantitative PCR.

Western blot analysis. The cells were harvested at $48 \mathrm{~h}$ after transfection, and lysed with RIPA buffer for $30 \mathrm{~min}$ at $4^{\circ} \mathrm{C}$. The protein concentration was determined using a BCA protein assay kit (Pierce, Rockford, IL, USA). Equal amounts of total protein of each treatment were separated using $12.5 \%$ SDS-PAGE according to Laemmli's method (12), and were then transferred onto polyvinylidene difluoride (PVDF) membranes. The first antibodies used were polyclonal mouse anti-CHPF (1:2,000 dilutions; Sigma-Aldrich ${ }^{\circledR}$ Co. LLC., St. Louis, MO, USA), and the anti-GAPDH antibody (1:2,000 dilution; Santa Cruz Biotechnology, Santa Cruz, CA, USA). The secondary antibody was anti-goat IgG-conjugated with horseradish peroxidase at a dilution of 1:2,000. The bound antibodies were detected using the Enhanced Chemiluminescence Plus Western Blotting Detection system (GE Healthcare, Bethesda, MD, USA). GAPDH was used as an internal control to normalize the CHPF expression levels.

Cell growth and MTT cell proliferation assays. Cell growth was assessed using multiparametric high-content screening (HCS). U251 cells at the logarithmic phase after being infected with either the NC or shRNA-CHPF lentiviruses were seeded at $2 \times 10^{3}$ cells/well in 96-well tissue culture plates, and incubated at $37^{\circ} \mathrm{C}$ for $1-5$ days. The cells in the plates were counted using the Cellomics ArrayScan ${ }^{\mathrm{TM}}$ VT1 HCS automated reader (Cellomics, Inc., Pittsburgh, PA, USA) for daily analysis . In each well, at least 800 cells were analyzed. All experiments were performed in triplicate.

In vitro cell proliferation was determined using an MTT cell proliferation assay kit as described by the manufacturer [American Type Culture Collection (ATCC) Manassas, VA, USA]. At the end of the culture period, the cells were washed with phosphate-buffered saline, the MTT reagents were then added according to the manufacturer's recommendations, and the absorbance was assessed at $570 \mathrm{~nm}$ using a microplate reader. Each experiment was repeated 3 times in duplicate.

Apoptosis and cell cycle analysis using flow cytometry. Annexin V-FITC/propidium iodide (PI) double labeling was used to determine the cell cycle distribution or detect apoptosis. U251 cells were infected with the shRNA-CHPF or NC plasmids and incubated at $37^{\circ} \mathrm{C}$. U251 cells were harvested by trypsinization and incubated with FITC-conjugated Annexin V and PI according to the manufacturer's instructions (KeyGen Biotech, Nanjing, China). FACSCalibur II sorter and Cell Quest Research Software (BD Biosciences, San Diego, CA, USA) were used for analysis. To further confirm the changes of DNA content distribution in the shRNA-CHPFtreated cells, we performed PI staining. The cells of different groups were collected and fixed in $70 \%$ ethanol at $4^{\circ} \mathrm{C}$ overnight. After incubation with $50 \mu \mathrm{g} / \mathrm{ml}$ of PI and RNase A (Sigma-Aldrich) for $30 \mathrm{~min}$ at $37^{\circ} \mathrm{C}$ in the dark, the cells were subjected to FACS analysis as described above. All experiments were performed in triplicate.

Statistical analysis. All statistical analyses were implemented in the SPSS 20.0 statistical software package. All data are presented as the means \pm standard deviations (SD) and the experiments were repeated 3 times. The Student's t-test was used for raw data analysis. A P-value of $<0.05$ was considered to indicate a statistically significant result.

\section{Results}

CHPF mRNA detection in 4 human glioma cell lines. The expression levels of CHPF mRNA were examined in glioma cell lines A172, U373, U251 and U87 by quantitative RT-PCR. The results revealed that CHPF mRNA was expressed in all 4 cell lines (Fig. 1).

CHPF is overexpressed in human glioma tissues. In order to determine whether CHPF was involved in the regulation 


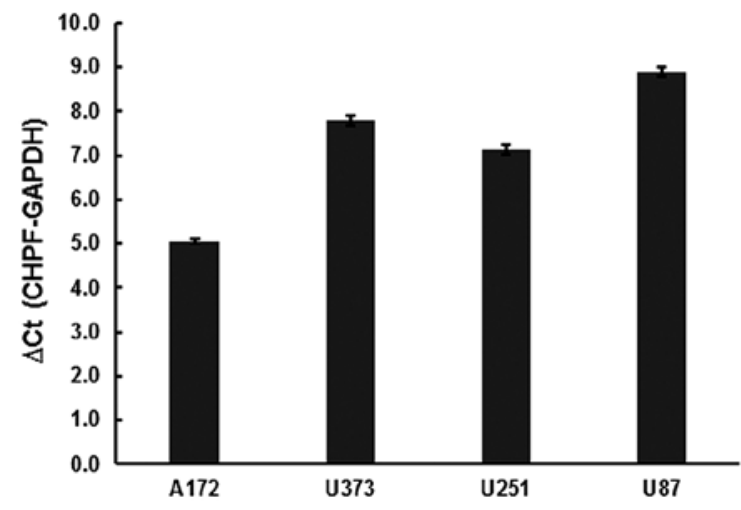

Figure 1. mRNA expression levels of CHPF in 4 glioma cell lines detected by quantitative RT-PCR. GAPDH was used as an internal control.CHPF, chondroitin polymerizing factor.

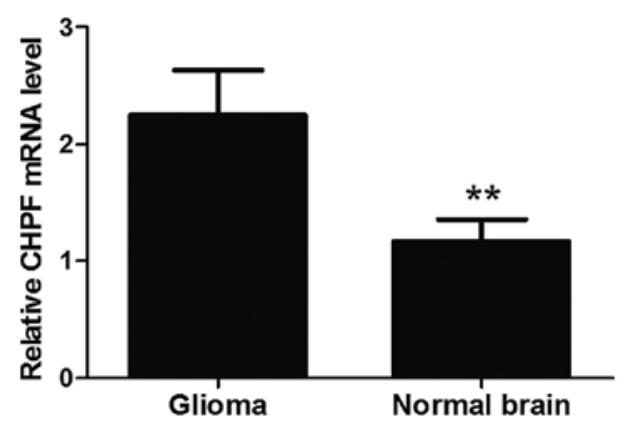

Figure 2. Expression of CHPF mRNA in resected gliomas and normal brain tissues. The expression of CHPF mRNA was detected by CHPF RT-PCR. GAPDH was used as an internal control; ${ }^{* *} \mathrm{P}<0.01$ vs. the normal group. $\mathrm{CHPF}$, chondroitin polymerizing factor.

of tumorigenesis of human glioma we assessed the CHPF expression level in glioma tissue specimens and normal brain tissues. Quantitative RT-PCR assays revealed that the mRNA levels of CHPF in gliomas tissues were significantly higher than those in normal brain tissues. The average expression level of CHPF in gliomas tissues was 2.484 and in normal brain tissues it was 1.231 ( $\mathrm{P}<0.01$; Fig. 2). This result demonstrated that CHPF was upregulated in glioma tissues compared with normal brain tissues.

Knockdown efficiency determined by western blot analysis. Human renal epithelial $293 \mathrm{~T}$ cells were infected with shRNA-CHPF or NC lentiviruses. The protein levels of CHPF were assessed using western blotting in $293 \mathrm{~T}$ cells. shRNA-CHPF-infected cultures had a significantly lower level of CHPF compared to the shRNA-Ctrl-infected cultures (Fig. 3).

Knockdown of CHPF by the shRNA lentiviral system in human glioma cell line U251. To explore the role of CHPF in glioma, shRNA-CHPF and shRNA-Ctrl expressing GFP were generated and infected into human glioma cell line U251. The concentration dose of the shRNA-CHPF used was $4 \times 10^{8} \mathrm{TU} / \mathrm{ml}$. More than $80 \%$ of the cells exhibited positive green fluorescence in both the shRNA-CHPF and shRNA-Ctrl groups, indicating the successful lentiviral infection (Fig. 4A). The knockdown effect was analyzed by qRT-PCR. At 5 days post-infection, the CHPF mRNA level of U251 cells infected

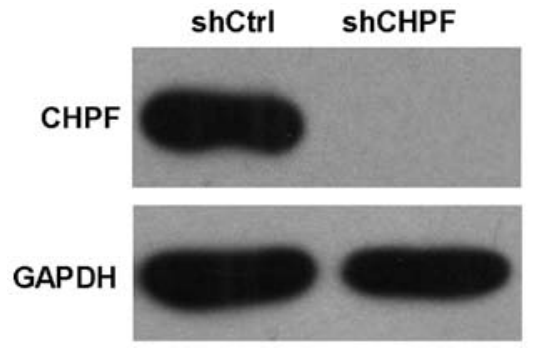

Figure 3. Expression of CHPF protein knockdown in 293T cells after transfection with the shRNA-CHPF (shCHPF) lentivirus or NC lentivirus. The protein expression of CHPF was analyzed by western blotting and GAPDH was used as a loading control. CHPF, chondroitin polymerizing factor; shCHPF, shRNA-CHPF; shCtrl, shRNA-Ctrl.

with shRNA-CHPF $(0.477 \pm 0.034)$ was significantly lower than

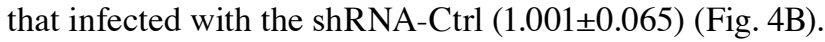

Knockdown of CHPF noticeably inhibits U251 cell growth. To examine the effect of CHPF on cell growth, U251 cells expressing either the shRNA-CHPF or shRNA-Ctrl were seeded into 96 -well plates and analyzed by Cellomics daily for 5 days. As shown in Fig. 5, the cell proliferation level in the shRNA-CHPF group remained lower from the second day after infection, and the gap between the shRNA-CHPF and shRNA-Ctrl groups increased with time. The results of the present study revealed that CHPF knockdown significantly inhibited the growth of the U251 cells.

To further evaluate the effect of CHPF on the regulation of glioma cell proliferation, an MTT assay was applied in U251 cells. As shown in Fig. 6, knockdown of CHPF expression markedly decreased the proliferation potential from day 2 $(\mathrm{P}<0.01)$. The growth of U251 cells treated with the shRNACHPF lentivirus was markedly inhibited compared to the shRNA-Ctrl group. The shRNA-CHPF cells had significantly decreased in vitro growth on days 4 (shCtrl, $5.64 \pm 0.04$ vs. shCHPF $2.96 \pm 0.05 ; \mathrm{P}<0.01)$ and 5 (shCtrl, $10.03 \pm 0.28$ vs. shCHPF, $3.67 \pm 0.07 ; \mathrm{P}<0.01)$. Based on these data, in vitro U251 cell growth was dependent on CHPF expression.

Inhibition of CHPF induces G0/G1 phase arrest in glioma U251 cells. To ascertain whether CHPF is necessary for cell cycle progression in U251 cells, we assessed the cell cycle phases in U251 cells by flow cytometry. As shown in Fig. 7, the shRNA-CHPF group displayed the following: G0/G1 phase, $65.57 \pm 0.42 \%$; $S$ phase, $18.48 \pm 0.26 \%$; and $\mathrm{G} 2 / \mathrm{M}$ phase, $15.95 \pm 0.32 \%$, and the shRNA-Ctrl group displayed the following distribution: G0/G1 phase, 53.42 $\pm 0.23 \%$; S phase, $30.49 \pm 0.70 \%$; and $\mathrm{G} 2 / \mathrm{M}$ phase, $16.10 \pm 0.50 \%$. With the absence of CHPF, the number of cells entering the G0/G1 phase increased by $22.7 \%(\mathrm{P}<0.05)$ and the cells entering the $\mathrm{S}$ phase decreased by $39.4 \%(\mathrm{P}<0.01)$. In conclusion, these data revealed that $\mathrm{CHPF}$ regulated $\mathrm{U} 251$ cell growth and blocked cell cycle progression in the $\mathrm{G} 0 / \mathrm{G} 1$ phase.

CHPF knockdown increases glioma U251 cell apoptosis. We further examined the effect of knockdown of CHPF on the cell apoptosis of glioma U251 cells by flow cytometry. The apoptosis percentage of U251 cells in the shRNA-CHPF group $(10.30 \pm 0.16) \%$ was significantly higher $(\mathrm{P}<0.05)$ than in the 

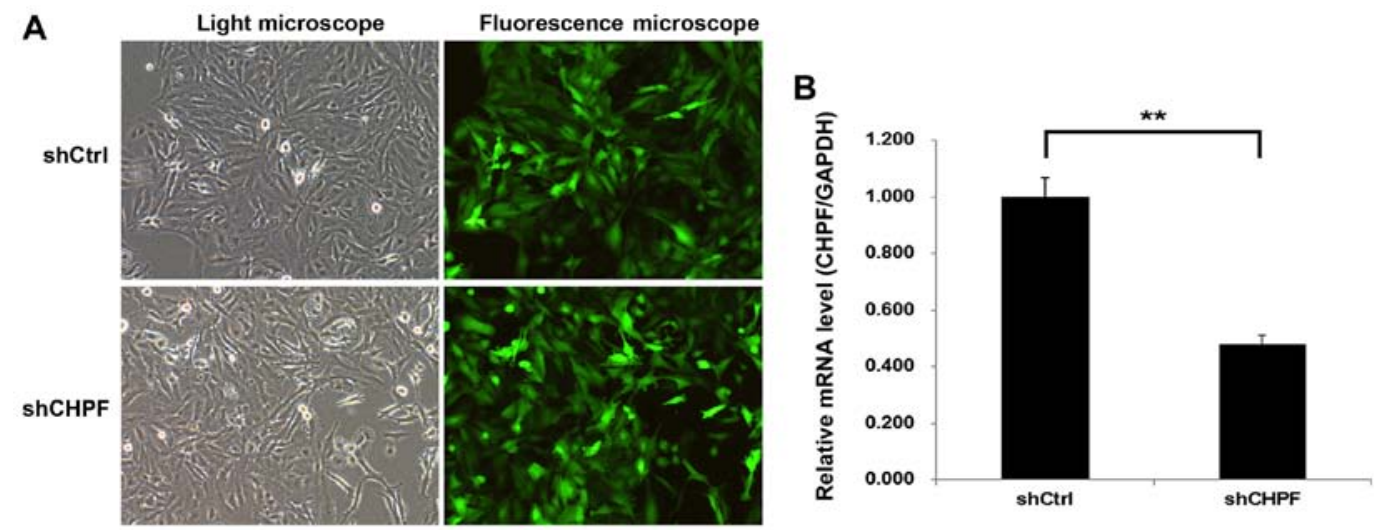

Figure 4. A lentiviral vector expressing shRNA-CHPF was constructed and transfected into the glioma U251 cells. (A) The infection efficiency was examined by fluorescence and light microscopy at $72 \mathrm{~h}$ post-infection. Most cells exhibited positive green fluorescence in both the shRNA-CHPF and shRNA-Ctrl groups. (B) The knockdown efficiency of CHPF was assessed by quantitative RT-PCR assay; ${ }^{* *} \mathrm{P}<0.01$. CHPF, chondroitin polymerizing factor; shCHPF, shRNA-CHPF; shCtrl, shRNA-Ctrl.
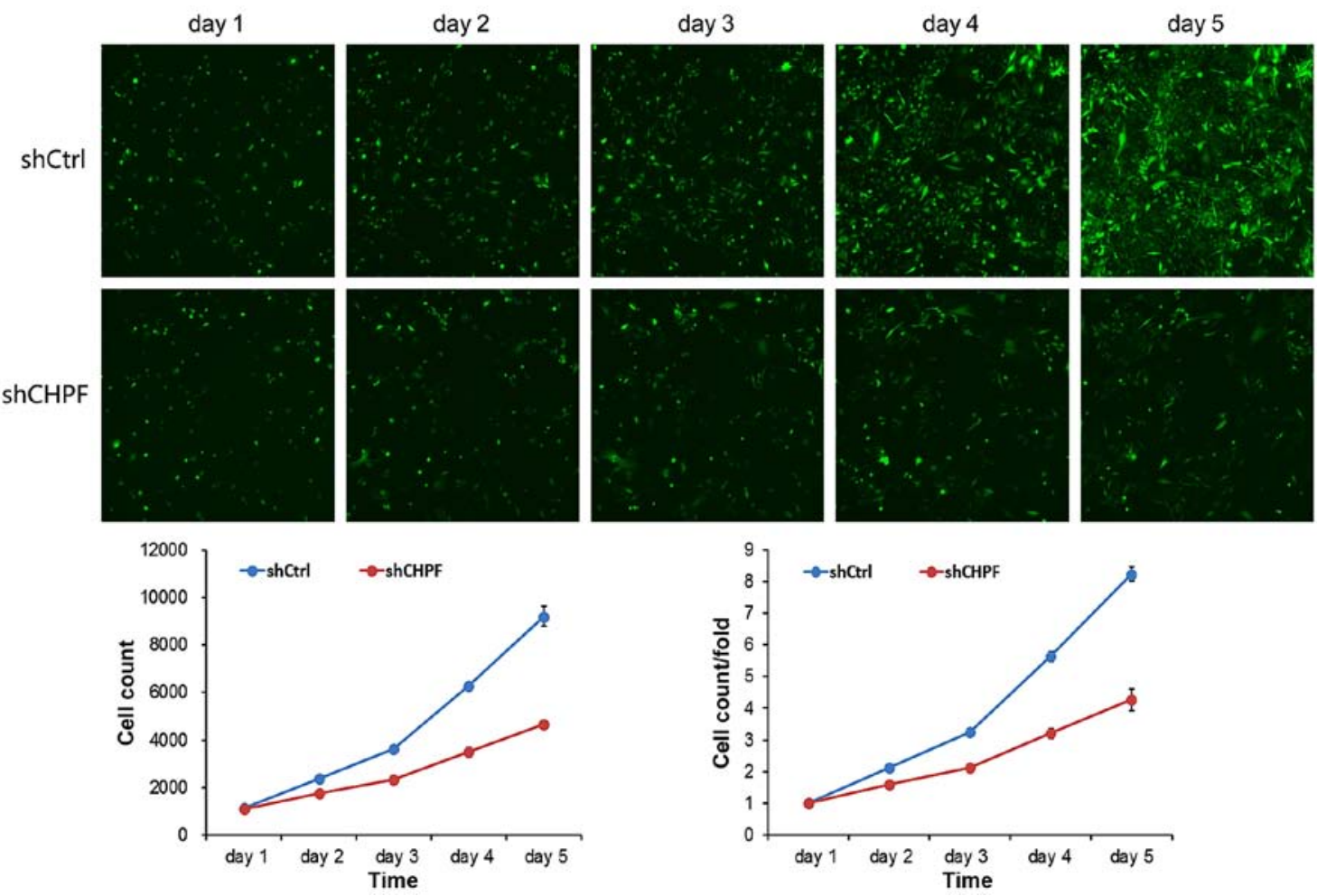

Figure 5. Knockdown of CHPF decreases the proliferation of glioma U251 cells. High-content cell imaging was applied as indicated daily to acquire raw images of cell growth after lentiviral infection. CHPF, chondroitin polymerizing factor; shCHPF, shRNA-CHPF; shCtrl, shRNA-Ctrl.
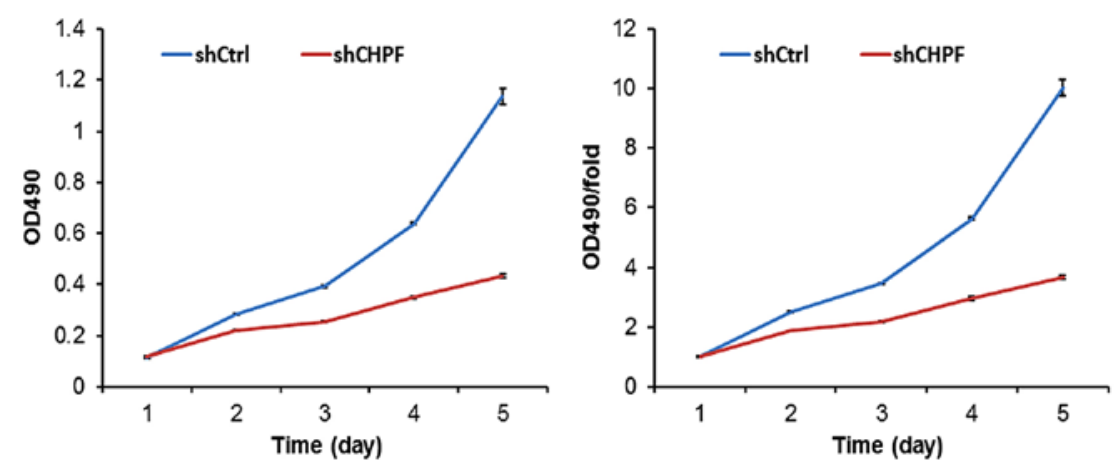

Figure 6. MTT assay displaying the proliferation ability of the U251 cells after transfection with shRNA-CHPF. Glioma U251 cells were treated with shRNA-CHPF or shRNA-Ctrl. CHPF, chondroitin polymerizing factor; shCHPF, shRNA-CHPF; shCtrl, shRNA-Ctrl. 

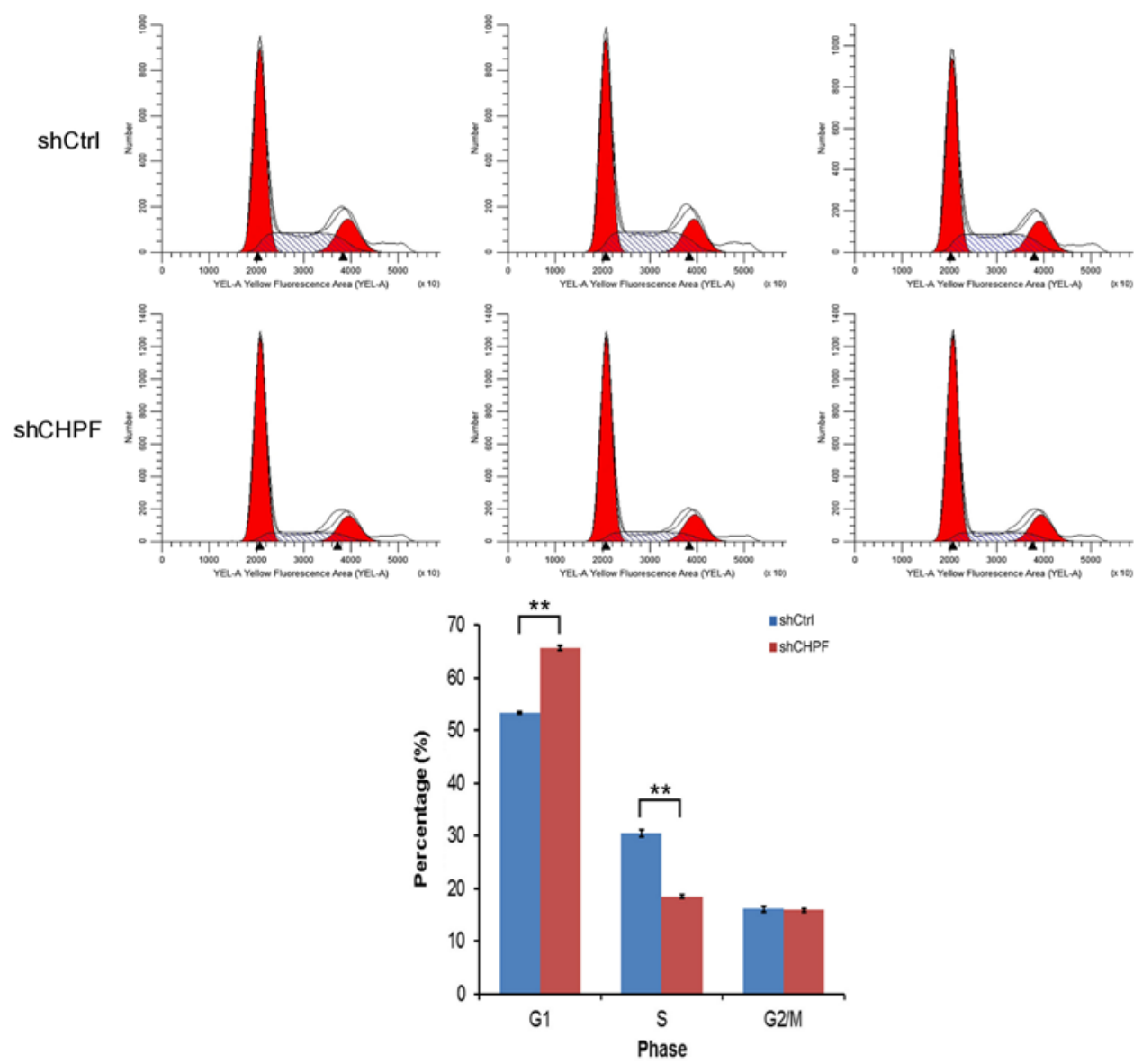

Figure 7. CHPF knockdown induces glioma U251 cell cycle arrest. Flow cytometry revealed the cell cycle distribution of the U251 cells after transfection with shRNA-CHPF. The experiments were performed in triplicate. ${ }^{* *} \mathrm{P}<0.01$ mean vs. the shRNA-Ctrl group. CHPF, chondroitin polymerizing factor; shCHPF, shRNA-CHPF; shCtrl, shRNA-Ctrl.
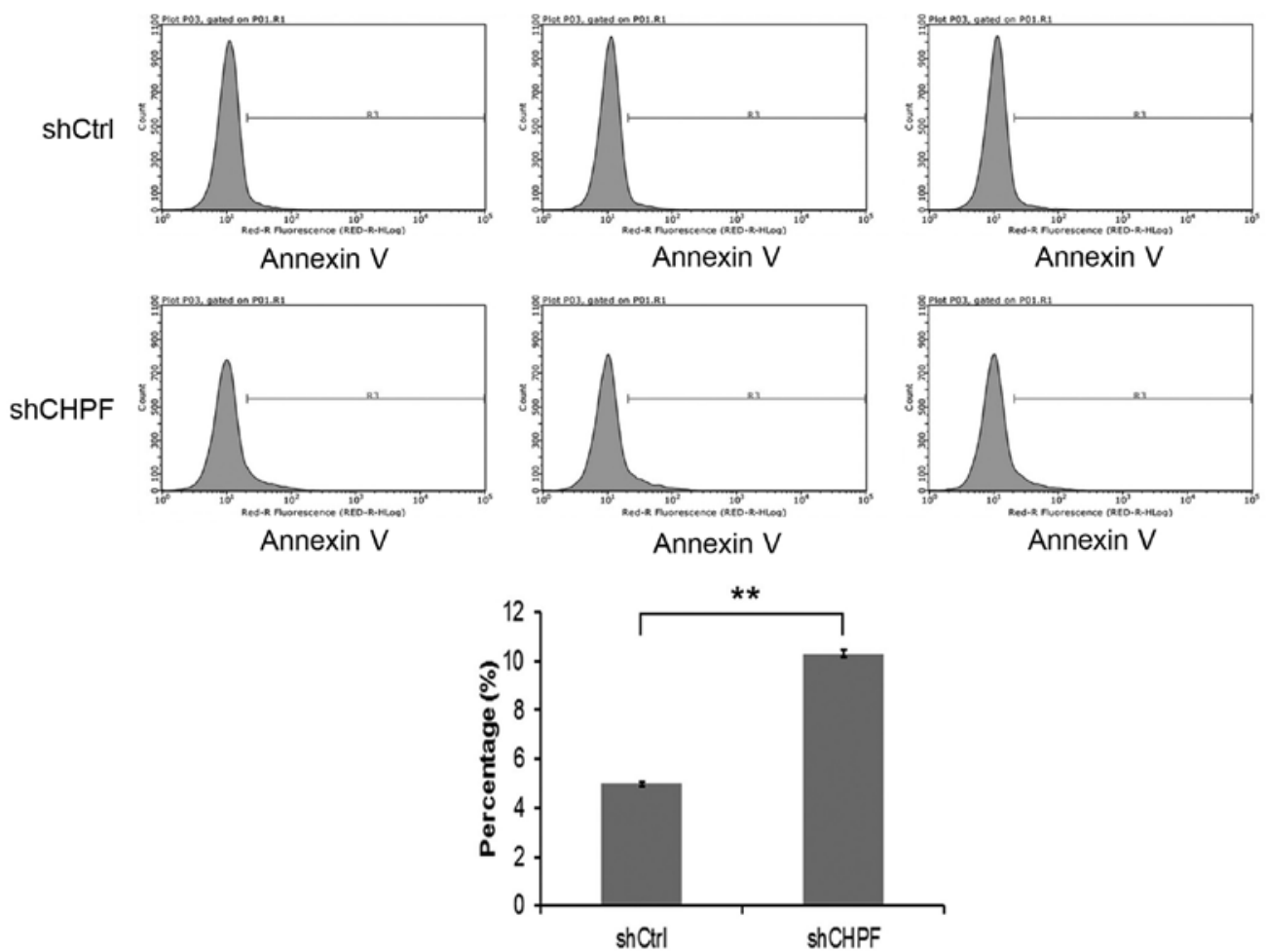

Figure 8. CHPF knockdown promotes glioma U251 cell apoptosis. The apoptotic rate of the U251 cells after transfection with shRNA-CHPF analyzed using flow cytometry. The experiments were performed in triplicate. ${ }^{* *} \mathrm{P}<0.01$ mean vs. the shRNA-Ctrl group. CHPF, chondroitin polymerizing factor; shCHPF, shRNA-CHPF; shCtrl, shRNA-Ctrl. 
shRNA-Ctrl group $(4.99 \pm 0.09) \%$ (Fig. 8). These results indicated that CHPF silencing inhibited the proliferation of U251 cells by inducing G0/G1 phase cell cycle arrest and apoptosis.

\section{Discussion}

Glioma, particularly glioblastoma, has a poor prognosis $(4,13)$. It is one of the most common highly vascularized malignant tumors of the CNS, with a $\leq 12$-month median survival period $(5,14)$. Therefore, it is essential to investigate the mechanisms involved in glioma initiation and progression.

Chondroitin sulfate (CS) is a polysaccharide consisting of repeating disaccharide units of $\mathrm{N}$-acetyl-D-galactosamine and D-glucuronic acid residues, modified with sulfated residues at various positions (11). CS biosynthesis and sulfation balance are tightly controlled and play important roles in the progression of the disease $(15,16)$. In addition, the specific sulfation pattern of CS chains dictates its function and binding affinities $(17,18)$. Previous studies utilizing inhibitors or enzymes which degrade CS chains indicate that CS has a considerable effect on the processes of tumor metastasis, proliferation and adhesion $(19,20)$. The identification of CHPF, a unique protein factor required for chondroitin polymerization activity, may shed light on the molecular basis of CS. Therefore, CHPF may play an important role in tumor progression. However, to date, only 3 studies have revealed the expression level of CHPF in cancer, including colorectal cancer (10), head and neck squamous cell carcinoma (21) and laryngeal cancer (22). To date, the function and the role of CHPF in human types of cancer remain unknown. Therefore, we employed LV-shRNA-CHPF to determine whether CHPF has effects on the progression of human glioma in vitro.

In the present study, we firstly demonstrated that CHPF was highly expressed in human glioma tissues and 4 glioma cell lines. To explore the role of CHPF in glioma, a lentiviral vector expressing CHPF shRNA was constructed and transfected into the glioma U251 cells, which stably downregulated the expression levels of the CHPF gene in U251 cells in vitro. Compared to the shRNA-Ctrl group of cells, the shRNA-CHPF group of cells exhibited decreased proliferation and a significant increase in the proportion of cells in the G0/G1 phase. In addition, we found that knockdown of the expression of CHPF increased apoptosis in glioma U251 cells. Therefore, our results confirmed that CHPF promotes glioma U251 cell growth. Further validation and functional evaluation are warranted to assess the role of CHPF in other glioma cell lines and the mechanism underlying this association.

In conclusion, our findings indicate that CHPF plays an important role in the progression of human glioma. A high level of expression of CHPF was found in human glioma tissues and 4 glioma cell lines. Downregulation of CHPF expression by the shRNA-CHPF lentiviral vector inhibited glioma U251 cell proliferation and induced cell apoptosis. This demonstrated that CHPF may be a novel target in the clinical treatment of gliomas.

\section{Acknowledgements}

The present study was supported by theNational Natural Science Foundation of China (grant no. 81660420), the Construction
Plan of the Superior Science and Technology Innovation Team of Jiangxi Province (grants no. 20152BCB24009), and the Foreign Science and Technology Cooperation Plan of Jiangxi Province (grants no. 20151BDH80009).

\section{References}

1. Wu M, Fan Y, Lv S, Xiao B, Ye M and Zhu X: Vincristine and temozolomide combined chemotherapy for the treatment of glioma: A comparison of solid lipid nanoparticles and nanostructured lipid carriers for dual drugs delivery. Drug Deliv 23: 2720-2725, 2016

2. Alves TR, Lima FR, Kahn SA, Lobo D, Dubois LG, Soletti R, Borges H and Neto VM: Glioblastoma cells: A heterogeneous and fatal tumor interacting with the parenchyma. Life Sci 89: 532-539, 2011.

3. Castro MG, Candolfi M, Kroeger K, King GD, Curtin JF, Yagiz K, Mineharu Y, Assi H, Wibowo M, Ghulam Muhammad AK, et al: Gene therapy and targeted toxins for glioma. Curr Gene Ther 11: 155-180, 2011.

4. Xiao B, Zhou X, Ye M, Lv S, Wu M, Liao C, Han L, Kang C and Zhu X: MicroRNA-566 modulates vascular endothelial growth factor by targeting Von Hippel-Landau in human glioblastoma in vitro and in vivo. Mol Med Rep 13: 379-385, 2016.

5. Fan YH, Ye MH, Wu L, Lv SG, Wu MJ, Xiao B, Liao CC, Ji QK, Chai Y and Zhu XG: Overexpression of miR-98 inhibits cell invasion in glioma cell lines via downregulation of IKKe. Eur Rev Med Pharmacol Sci 19: 3593-3604, 2015.

6. Wu L, Yang L, Xiong Y, Guo H, Shen X, Cheng Z, Zhang Y, Gao $Z$ and $Z$ hu $X$ : Annexin A5 promotes invasion and chemoresistance to temozolomide in glioblastoma multiforme cells. Tumour Biol 35: 12327-12337, 2014.

7. Kitagawa H, Izumikawa T, Uyama T and Sugahara K: Molecular cloning of a chondroitin polymerizing factor that cooperates with chondroitin synthase for chondroitin polymerization. J Biol Chem 278: 23666-23671, 2003.

8. Kalathas D, Theocharis DA, Bounias D, Kyriakopoulou D, Papageorgakopoulou N, Stavropoulos MS and Vynios DH: Alterations of glycosaminoglycan disaccharide content and composition in colorectal cancer: Structural and expressional studies. Oncol Rep 22: 369-375, 2009.

9. Pothacharoen P, Siriaunkgul S, Ong-Chai S, Supabandhu J, Kumja P, Wanaphirak C, Sugahara K, Hardingham T and Kongtawelert P: Raised serum chondroitin sulfate epitope level in ovarian epithelial cancer. J Biochem 140: 517-524, 2006.

10. Kalathas D, Theocharis DA, Bounias D, Kyriakopoulou D, Papageorgakopoulou N, Stavropoulos MS and Vynios DH: Chondroitin synthases I, II, III and chondroitin sulfate glucuronyltransferase expression in colorectal cancer. Mol Med Rep 4: 363-368, 2011.

11. Ogawa H, Shionyu M, Sugiura N, Hatano S, Nagai N, Kubota Y, Nishiwaki K, Sato T, Gotoh M, Narimatsu H, et al: Chondroitin sulfate synthase-2/chondroitin polymerizing factor has two variants with distinct function. J Biol Chem 285: 34155-34167, 2010.

12. Liu H, Liang S, Yang X, Ji Z, Zhao W, Ye X and Rui J: RNAi-mediated RPL34 knockdown suppresses the growth of human gastric cancer cells. Oncol Rep 34: 2267-2272, 2015.

13. Ivo D'Urso P, Fernando D'Urso O, Damiano Gianfreda C, Mezzolla V, Storelli C and Marsigliante S: miR-15b and miR-21 as circulating biomarkers for diagnosis of glioma. Curr Genomics 16: 304-311, 2015.

14. Viswanath P and Ronen SM: Metabolic reprogramming of pyruvate dehydrogenase is essential for the proliferation of glioma cells expressing mutant IDH1. Mol Cell Oncol 3: e1077922, 2016.

15. Prinz RD, Willis CM, van Kuppevelt TH and Klüppel M: Biphasic role of chondroitin sulfate in cardiac differentiation of embryonic stem cells through inhibition of Wnt/ $\beta$-catenin signaling. PLoS One 9: e92381, 2014.

16. Willis CM and Klüppel M: Chondroitin sulfate-E is a negative regulator of a pro-tumorigenic Wnt/beta-catenin-Collagen 1 axis in breast cancer cells. PLoS One 9: e103966, 2014.

17. Klüppel M: The roles of chondroitin-4-sulfotransferase-1 in development and disease. Prog Mol Biol Transl Sci 93: 113-132, 2010. 
18. Karangelis DE, Kanakis I, Asimakopoulou AP, Karousou E Passi A, Theocharis AD, Triposkiadis F, Tsilimingas NB and Karamanos NK: Glycosaminoglycans as key molecules in atherosclerosis: The role of versican and hyaluronan. Curr Med Chem 17: 4018-4026, 2010.

19. Fthenou E, Zong F, Zafiropoulos A, Dobra K, Hjerpe A and Tzanakakis GN: Chondroitin sulfate A regulates fibrosarcoma cell adhesion, motility and migration through JNK and tyrosine kinase signaling pathways. In Vivo 23: 69-76, 2009.

20. Denholm EM, Lin YQ and Silver PJ: Anti-tumor activities of chondroitinase AC and chondroitinase B: Inhibition of angiogenesis, proliferation and invasion. Eur J Pharmacol 416: 213-221, 2001.
21. Teh MT, Gemenetzidis E, Patel D, Tariq R, Nadir A, Bahta AW, Waseem A and Hutchison IL: FOXM1 induces a global methylation signature that mimics the cancer epigenome in head and neck squamous cell carcinoma. PLoS One 7: e34329, 2012.

22. Kalathas D, Triantaphyllidou IE, Mastronikolis NS, Goumas PD, Papadas TA, Tsiropoulos G and Vynios DH: The chondroitin/dermatan sulfate synthesizing and modifying enzymes in laryngeal cancer: Expressional and epigenetic studies. Head Neck Oncol 2: 27, 2010. 